\title{
Compressive Sampling Orthogonal Matching Pursuit Algorithm Based on Peak Signal to Noise Ratio
}

\author{
Hu $\operatorname{Dan}^{1}$ \\ ${ }^{1}$ College of Big Data and Information Engineering, Guizhou University, \\ Guiyang Guizhou, 550025, China
}

\begin{abstract}
In order to improve signal reconstruction accuracy, a CoSaMP (Compressive Sampling Matching Pursuit) algorithm based on peak signal to noise ratio is proposed in allusion to the disadvantages of CoSaMP algorithm. Firstly, the discrete cosine wave transform is improved to initially estimate the signal sparseness; secondly, the optimum iteration number is determined according to the peak signal to noise ratio to gradually approach to the real sparseness of the signal for signal reconstruction; finally, the simulation experiment is adopted to analyze the algorithm performance. The result shows: compared with CoSaMP algorithm and other improved CoSaMP algorithms, the proposed algorithm can not only obtain more ideal reconstruction effect, but also improve the reconstruction success probability and reduce the reconstruction time, thus having higher reconstruction efficiency.
\end{abstract}

Keywords: Compressive Sensing; Matching Pursuit; Sparse Estimation; Signal Reconstruction; Discrete Cosine Wave Transform

\section{Introduction}

Due to the condition limitation during signal acquisition process, it is difficult to acquire complete data. Therefore, the recovery of the original signal from the incomplete data has important practical value [1]. As a new signal processing technology, CS (Compressive Sensing) can be used to sparsely represent and accurately reconstruct the signals at a sampling rate much lower than Nyquist sampling rate and has important application value in such fields as signal processing, wireless communication, geological prospecting, medical imaging and image processing, thus becoming an important research subject at present [2].

CS theory has three core issues: sparse transform of signal, observation matrix design and reconstruction algorithm, wherein the reconstruction algorithm as one of the key technologies of CS theory [3] determines whether CS theory is practical and feasible. Existing CS reconstruction algorithms include two types: convex optimization algorithm and greedy algorithm [4], wherein the convex optimization algorithm mainly includes interior point method and threshold value method, and aims at adopting minimization algorithm to estimate the signal in order to recover the original signal [5]; the greedy algorithm mainly includes MP (Matching Pursuit) algorithm and OMP (Orthogonal Matching Pursuit) algorithm [6]. In the greedy reconstruction algorithms, the selection of the support set is mainly optimized, and these algorithms have some disadvantages [7]. Therefore, SP (Subspace Pursuit) algorithm with strong robustness is proposed in literature [8], and afterwards CoSaMP (Compressive Sampling Matching Pursuit) algorithm is proposed in literature [9], but CoSaMP algorithm needs prior information in practical application, so the practical application scope thereof is limited [10].

In order to solve the problems existing in CoSaMP algorithm, a CoSaMP algorithm based on PSNR (Peak Signal to Noise Ratio) is proposed in this article, and the simulation experiment is carried out for analyzing the algorithm performance. 


\section{Compressive Sensing Theory}

$N \times 1$ digital signal $x$ is assumed in this article and it is non-sparse under natural condition. A suitable sparse transform matrix $\psi \in R^{N \times N}$ is adopted to convert signal $x$ into $K$ sparse signal $f$ :

$$
f=\psi x
$$

According to CS theory, if the measurement matrix $\varphi \in R^{M \times N}(M<<N)$ and the projection $y$ of the unknown signal in the measurement matrix $\varphi$ are known:

$$
y=\varphi f
$$

Then, in consideration of reconstruction, since the number of the equations is less than the number of the unknown parameters, this equation is an ill-condition equation and has

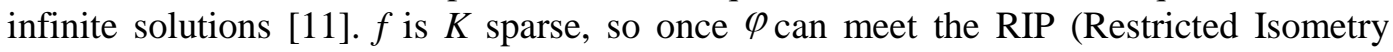
Property) condition, signal $f$ can be accurately reconstructed through adopting y to solve l0-norm problem according to relevant theoretical proof.

$$
\begin{aligned}
& \widehat{f}=\arg \min \|f\|_{0} \\
& \text { s.t. } \\
& \varphi f=y
\end{aligned}
$$

Similarly, if the sparse process and the measurement process are combined as follows:

$$
y=\varphi \psi x=\tilde{\varphi} x
$$

Then, since $\psi$ is fixed, once $\varphi$ meets certain conditions to enable $\tilde{\varphi}$ to meet RIP principle, signal $\mathrm{x}$ can be accurately reconstructed through solving $l 0$-norm problem:

$$
\begin{aligned}
& \hat{x}=\arg \min \|x\|_{0} \\
& \text { s.t. } \\
& \tilde{\varphi} x=y
\end{aligned}
$$

In conclusion, CS based reconstruction principle is as shown in Figure 1.

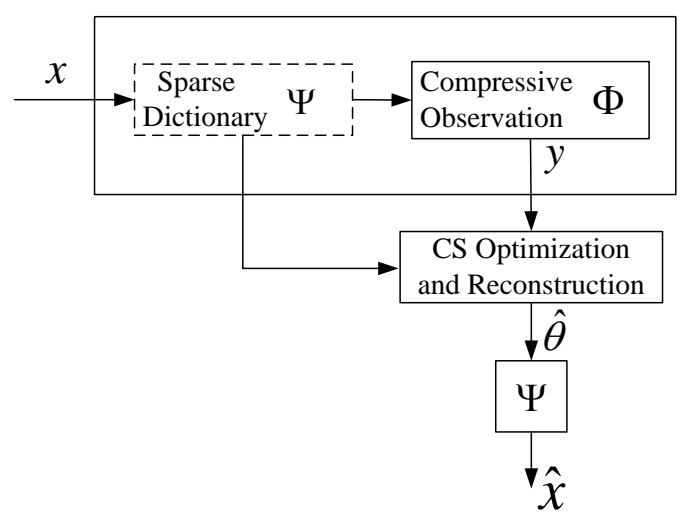

Figure 1. CS based Reconstruction Block Diagram

\section{Improved CoSaMP Algorithm}

\subsection{CoSaMP Algorithm}

CoSaMP algorithm is a greedy algorithm, and the objective function solved thereby is as follows:

$$
\hat{\mathbf{a}}=\min \|\mathbf{a}\|_{0}, \text { s.t. } \mathbf{y}=\mathbf{\Phi} \mathbf{x}=\boldsymbol{\Phi} \Psi \mathbf{a}
$$

In the above formula, $\mid \mathbf{a} \|_{0}$ represents the number of nonzero elements in vector $\mathbf{a}$ [12]. CoSaMP algorithm steps are as follows: 
(1) Initialize the allowance $\mathbf{r}^{0}=\mathbf{y}$ and estimate the sparseness (assumed as $\mathrm{K}$ ) of the signal, wherein the sets are set as $\Gamma^{0}=\varnothing$ and $J=\varnothing$ when the iteration number is $\mathrm{n}=1$;

(2) Calculate the signal agent: $\mathbf{c}=\boldsymbol{\Phi}^{T} \mathbf{r}_{n-1}=\left\langle\boldsymbol{\Phi}^{T}, \mathbf{r}_{n-1}\right\rangle$;

(3) Select $2 K$ elements with maximum energy in $\mathrm{c}$, wherein the set composed of the corresponding index value sequences is called $J$ index set $\Gamma^{n}=\Gamma^{n-1} \cup\{J\}$, and the corresponding atoms form the support set $\boldsymbol{\Phi}_{\Gamma^{n}}$;

(4) Adopt the least square method to update the approximate value of the signal to obtain $\hat{\mathbf{x}}$, then clip $\hat{\mathbf{x}}$ and select the index values corresponding to $K$ maximum values, wherein the set composed of these index values is set as $F^{n}$, then calculate $\hat{\mathbf{x}}$ and update the allowance $\mathbf{r}^{n}=\mathbf{y}-\boldsymbol{\Phi}_{F^{n}} \hat{\mathbf{x}}$;

(5) If the iteration termination condition can be met, output $\hat{\mathbf{x}}$; or else, return to Step (2);

In the algorithm, $\left\langle\boldsymbol{\Phi}^{T}, \mathbf{r}_{n-1}\right\rangle$ represents the inner product of $\mathbf{r}_{n-1}$ and each column in $A$, and the least square method is adopted to update the approximate value $\mathbf{X}^{n}=\left(\boldsymbol{\Phi}_{\Gamma^{n}}^{T} \boldsymbol{\Phi}_{\Gamma^{n}}\right)^{-1} \boldsymbol{\Phi}_{\Gamma^{n}}^{T} \mathbf{y}$ of the signal. Additionally, the iteration termination condition of the algorithm can be described as follows:

$$
\begin{array}{cr} 
& \left\|\mathbf{x}-\hat{\mathbf{x}}_{n+1}\right\|_{2} \leq 0.5\left\|\mathbf{x}-\hat{\mathbf{x}}_{n}\right\|_{2} \\
\text { or } & \mid \mathbf{x}-\hat{\mathbf{x}}_{n+1}\left\|_{2} \leq 2^{-n}\right\| \mathbf{x} \|_{2}
\end{array}
$$

\subsection{Improvement of CoSaMP Algorithm}

CoSaMP algorithm needs to take the signal sparseness as the prior information, but the signal sparseness is usually unknown in practical problems, so CoSaMP algorithm has many disadvantages. Therefore, the improved CoSaMP algorithm is adopted in this article [13].

\subsubsection{Improved Discrete Cosine Transform}

If only a few data of a signal are nonzero, then such signal is called as sparse signal. Among natural signals, only a few signals are sparse, but non-sparse signals can be converted into sparse signals through certain transforms. Specifically, DCT (Discrete Cosine Transform) is a common sparse signal processing technology, and DCT basis can be constructed by the following function:

$$
g(x, u)=\sqrt{\frac{2}{N}} \cos \frac{(2 x+1) u \pi}{2 N}
$$

In the above formula, $N$ is the lateral resolution of the image; $x=0,1 \ldots N-1 ; u=0,1 \ldots N-$ 1.

DCT basis constructed by Formula (8) has fixed form, but signals have multiple forms, so such single-form DCT basis cannot meet the sparseness requirements of all signals. Therefore, DCT basis is correspondingly improved as follows in this article:

$$
g(x, u)=\cos \left(\frac{x u \pi}{p}-\frac{q \pi}{10}\right)
$$

In the above formula, parameters $p$ and $q$ respectively represent the adjustment frequency and the phase; $x=0,1 \ldots N-1 ; u=0,1 \ldots N-1 ; p=N-20 \ldots N+20 ; q=1,2 \ldots 20 ; \mathrm{N}$ represents the lateral resolution of the signal.

\subsubsection{PSNR Value of Reconstructed Signal}

$X 0$ represents the original signal; after $K$ iterations, the PSNR value of the reconstructed signal is as follows: 


$$
\operatorname{PSNR}(K)=20 \lg \frac{255^{2}}{\left\|\widehat{X}_{K}-X_{0}\right\|_{2}^{2}}
$$

In practical application process, it is difficult to obtain the original signal, so Formula (10) cannot be adopted to calculate PSNR values. However, the observed value of the signal can be obtained, and Formula (10) is accordingly changed as follows:

$$
\operatorname{PSNR}(K) \approx 20 \lg \frac{255^{2}}{\left\|A^{*} X_{K}-Y\right\|_{2}^{2}}
$$

In the above formula, matrix $A$ is the observation matrix able to meet $s$-order RIP condition; $Y$ is the observed value.

Therefore, the optimal iteration number can be determined according to PSNR value in order to gradually approach to the real sparseness for signal reconstruction.

\section{Simulation Experiment}

\subsection{Simulation Environment}

In order to test the performance of the proposed algorithm, MATLAB R2010b is adopted for the simulation experiment in the computer with the configurations as Pentium(R) 4-core $2.8 \mathrm{GHz}$, 8G RAM, Windows 7 operating system. Meanwhile, CoSaMP algorithm and the algorithm proposed in literature [15] are adopted as the comparison algorithms in the experiment. The simulation objects are four standard gray images: "Lena", "Cameraman", "Barbara" and "Baboons", as shown in Figure 2, wherein the images are zoomed to $256 \times 256[16]$.

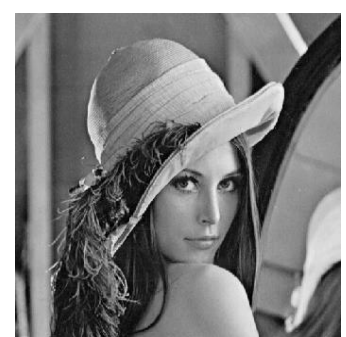

(a) Lena Image

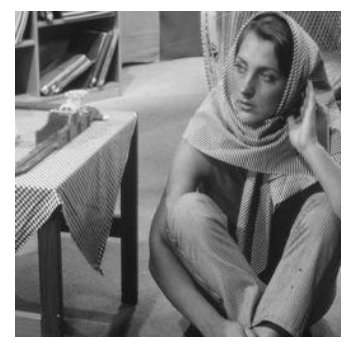

(c) Barbara

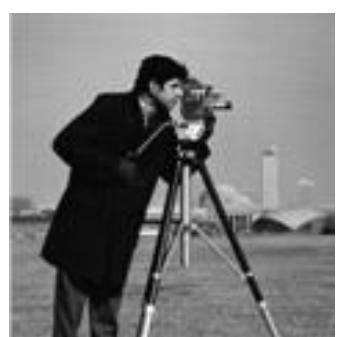

(b) Cameraman

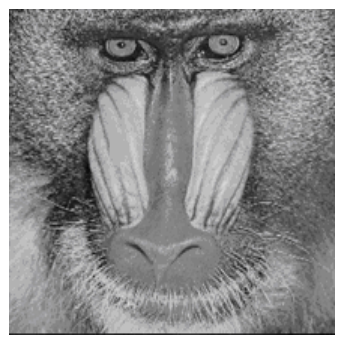

(d) Baboons

\section{Figure 2. Simulation Object}

\subsection{Result Analysis}

4.2.1. Comparison of the Visual Quality of the Reconstruction Results of Different Algorithms: The reconstruction results of CoSaMP algorithm, the algorithm in literature [15] and the proposed algorithm are as shown in Figures 3 5. According to Figures 3 5, the image reconstruction effects of these algorithms are significantly different from each other, wherein the proposed algorithm can obtain relatively perfect reconstructed image and the reconstruction effect thereof is obviously superior to those of other algorithms. 


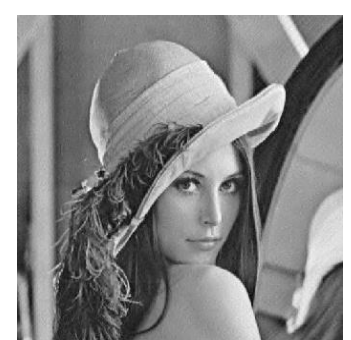

(a) Lena Image

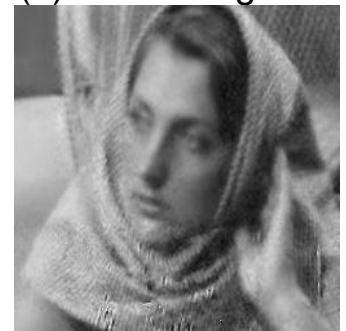

(c) Barbara

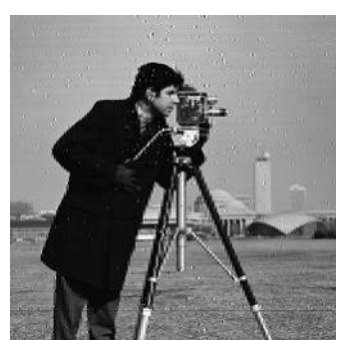

(b) Cameraman

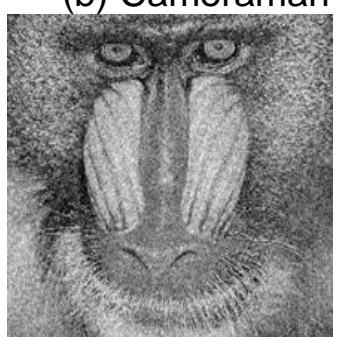

(d) Baboons

Figure 3. Reconstruction Effect of CoSaMP Algorithm

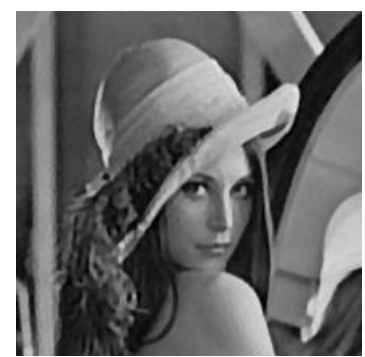

(a) Lena image

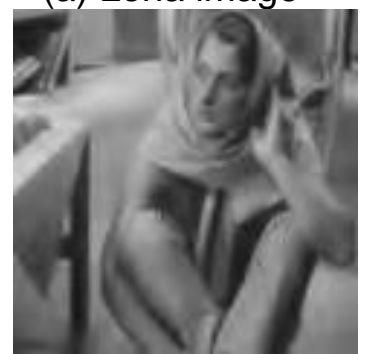

(c) Barbara

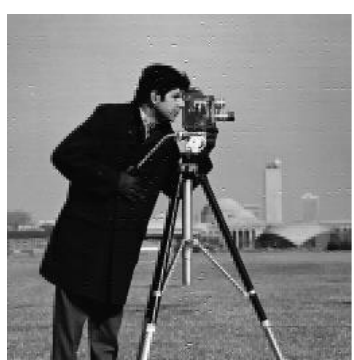

(b) Cameraman

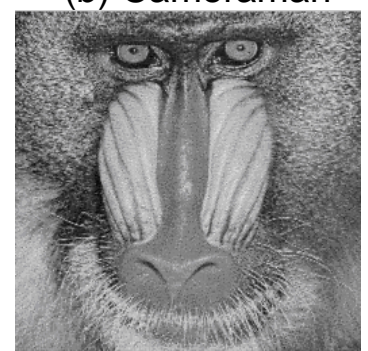

(d) Baboons

Figure 4. Reconstruction Effect of algorlthm in Literature [15]

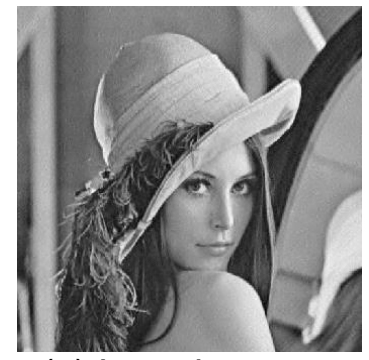

(a) Lena image

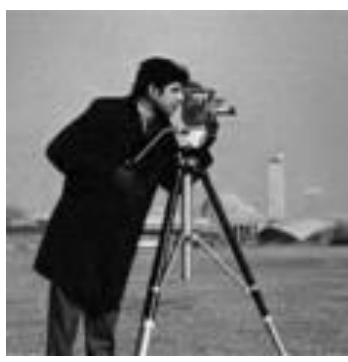

(b) Cameraman 


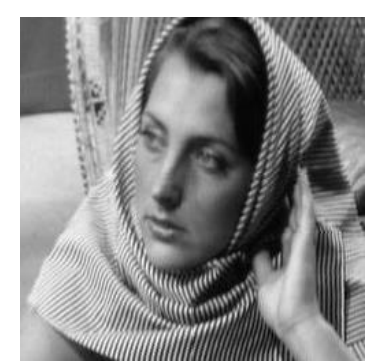

(c) Barbara

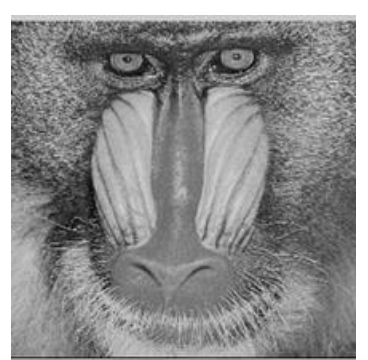

(d) Baboons

Figure 5. Reconstruction Effect of the Proposed Algorithm

4.2.2. Analysis of Reconstruction Effects under Different Sampling Rates: In order to test the influence of different sampling rates on the reconstruction effect of the proposed algorithm, the sampling rate for Lena image is set in the range of 20\% 50\% and the corresponding reconstruction effect is as shown in Figure 6. According to Figure 6, the reconstruction effect is improved along with the increase of the sampling rate, because more observed values make the image measurement more accurate. Meanwhile, the proposed algorithm also can obtain relatively ideal reconstruction effect at a very low sampling rate.
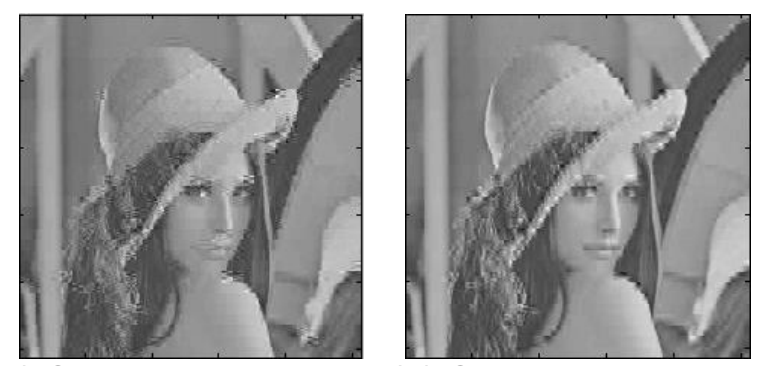

(a) Sampling rate $=20 \%$
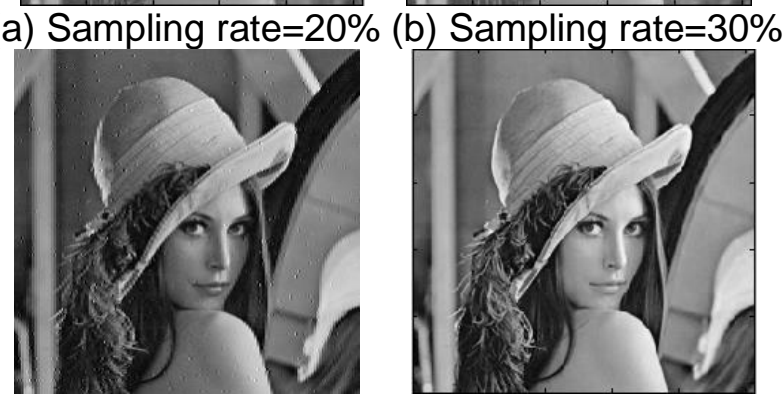

(a) Sampling rate $=40 \%$

(b) Sampling rate $=50 \%$

Figure 6. Images Reconstructed by the Proposed Algorithm under Different Sampling Rates

The sampling rate based PSNR change curves of CoSaMP algorithm, the algorithm in literature [15] and the proposed algorithm are as shown in Figure 7. According to Figure 7, PSNR value of the image reconstructed by the proposed algorithm is approximately linearly improved and the proposed algorithm is obviously superior to other algorithms in the aspect of reconstruction performance. 


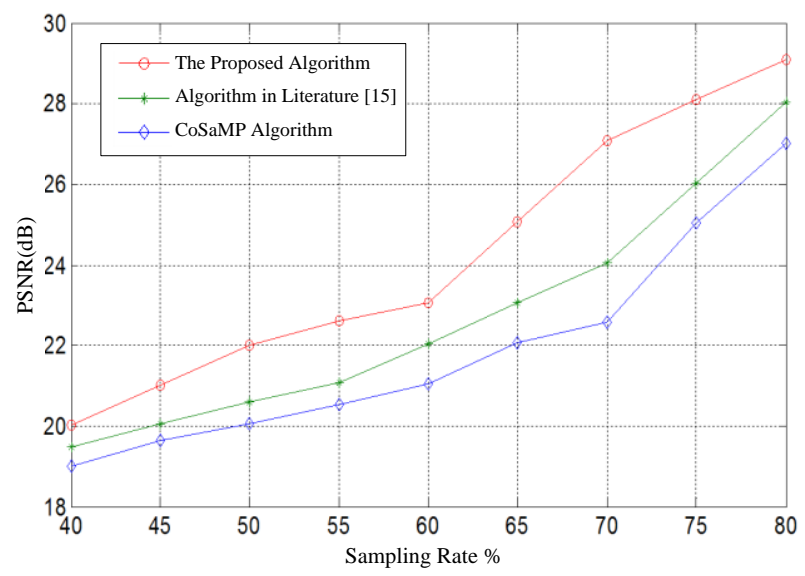

Figure 7. Sampling Rate based PSNR Change Curve

4.2.3. Comparison of reconstruction success probabilities of different algorithms: The absolutely sparse 0-1 random signals are sampled at the same sampling (40\%) for reconstruction, and the change relation between the sparseness and the reconstruction success probability is as shown in Figure 8. According to Figure 8, the signal reconstruction success rate probability is increased along with the increase of the sparseness. Under the same sparseness, the proposed algorithm is superior to the comparison algorithms in the aspect of reconstruction success probability.

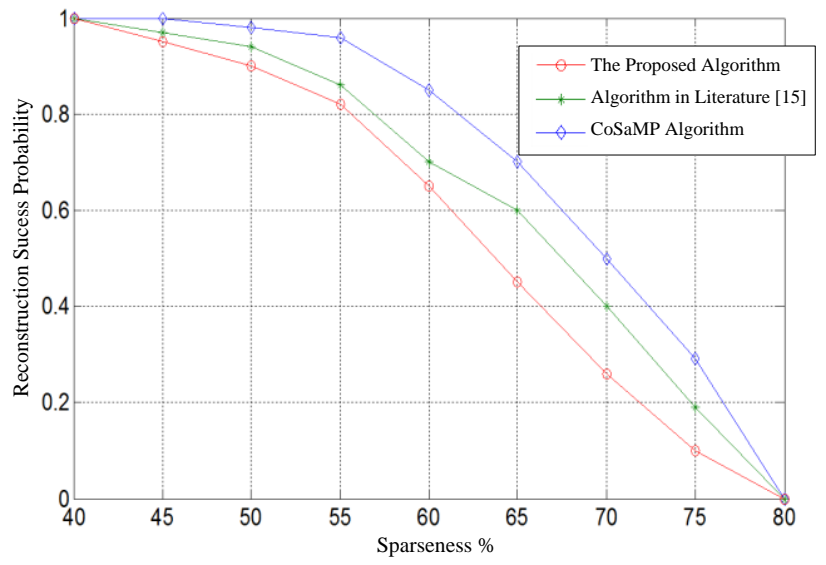

Figure 8. Relation between Sparseness and Reconstruction Sucess Probability

4.2.4. Comparison of reconstruction time of different algorithms: The reconstruction time can reflect the reconstruction speed of the algorithm, so the corresponding simulation experiment is carried out for 20 times for testing the reconstruction time of the algorithms, and the mean reconstruction time is adopted as the final result. The reconstruction time (unit: s) of the algorithms is as shown in Figure 9. According to Figure 9, compared with the comparison algorithms, the proposed algorithm has shortest mean reconstruction time and maximum reconstruction efficiency. 


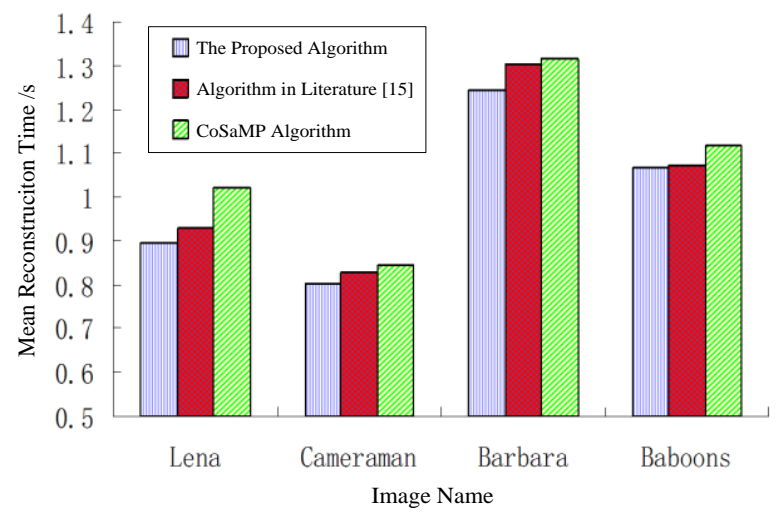

Figure 9. Comparison of Reconstruction Time of Different Algorithms

\section{Conclusion}

In order to obtain more ideal reconstruction result, CoSaMP algorithm based on PSNR is proposed in this article. Firstly, the improved cosine wave transform is adopted for signal processing; secondly, the recursion thought is used to gradually approach to the real sparseness of the signal in order to reconstruct the signal. The simulation experiment result shows that the proposed algorithm can not only obtain more ideal reconstruction result and increase the reconstruction success probability, but also improve the reconstruction accuracy and the operation efficiency, thus verifying that the proposed algorithm is obviously superior to other signal reconstruction algorithms in the aspect of reconstruction quality.

\section{Acknowledgement}

The paper is supported by the project of "Study on linear block code of blind identification based on Orthogonal Matching Pursuit(QIAN Ke He LH Zi[2014]7627)"

\section{References}

[1] J. Hu and Z. Gao, "Distinction immune genes of hepatitis-induced heptatocellular carcinoma", Bioinformatics, vol. 28, no. 24, (2012), pp. 3191-3194.

[2] J. Yang, B. Chen and J. A. Zho, "A Low-Power and Portable Biomedical Device for Respiratory Monitoring with a Stable Power Source", Sensors, vol. 15, no. 8, (2015), pp. 19618-19632.

[3] G. Bao, L. Mi, Y. Geng and K. Pahlavan, "A computer vision based speed estimation technique for localizing the wireless capsule endoscope inside small intestine", 36th Annual International Conference of the IEEE Engineering in Medicine and Biology Society (EMBC), (2014).

[4] X. Song and Y. Geng, "Distributed community detection optimization algorithm for complex networks", Journal of Networks, vol. 9, no. 10, (2014), pp. 2758-2765.

[5] D. Jiang, X. Ying and Y. Han, "Collaborative multi-hop routing in cognitive wireless networks", Wireless Personal Communications, (2015), pp. 1-23.

[6] Z. Lv, A. Halawani and S. Feng, "Multimodal hand and foot gesture interaction for handheld devices", ACM Transactions on Multimedia Computing, Communications, and Applications (TOMM), vol. 11, no. 1 s, (2014), p. 10.

[7] G. Liu, Y. Geng and K. Pahlavan, "Effects of calibration RFID tags on performance of inertial navigation in indoor environment", 2015 International Conference on Computing, Networking and Communications (ICNC), (2015).

[8] J. He, Y. Geng, Y. Wan, S. Li and K. Pahlavan, "A cyber physical test-bed for virtualization of RF access environment for body sensor network”, IEEE Sensor Journal, vol. 13, no. 10, (2013), pp. 38263836.

[9] W. Huang and Y. Geng, "Identification Method of Attack Path Based on Immune Intrusion Detection", Journal of Networks, vol. 9, no. 4, (2014), pp. 964-971.

[10] X. Li, Z. Lv and J. Hu, "XEarth: A 3D GIS Platform for managing massive city information", Computational Intelligence and Virtual Environments for Measurement Systems and Applications (CIVEMSA), 2015 IEEE International Conference on. IEEE, (2015), pp. 1-6. 
[11] J. He, Y. Geng, F. Liu and C. Xu, "CC-KF: Enhanced TOA Performance in Multipath and NLOS Indoor Extreme Environment”, IEEE Sensor Journal, vol. 14, no. 11, (2014), pp. 3766-3774.

[12] N. Lu, C. Lu, Z. Yang and Y. Geng, "Modeling Framework for Mining Lifecycle Management, Journal of Networks, vol. 9, no. 3, (2014), pp. 719-725.

[13] K. Wang, X. Zhou and T. Li, "Optimizing load balancing and data-locality with data-aware scheduling", Big Data (Big Data), 2014 IEEE International Conference on. IEEE, (2014), 119-128.

[14] L. Zhang, B. He and J. Sun, "Double Image Multi-Encryption Algorithm Based on Fractional Chaotic Time Series", Journal of Computational and Theoretical Nanoscience, vol. 12, (2015), pp. 1-7.

[15] T. Su, Z. Lv and S. Gao, "3d seabed: 3d modeling and visualization platform for the seabed", Multimedia and Expo Workshops (ICMEW), 2014 IEEE International Conference on IEEE, (2014), pp. $1-6$.

[16] Y. Geng, J. Chen, R. Fu, G. Bao and K. Pahlavan, "Enlighten wearable physiological monitoring systems: On-body rf characteristics based human motion classification using a support vector machine, IEEE transactions on mobile computing, vol. 1, no. 1, (2015), pp. 1-15.

[17] Y. Geng and K. Pahlavan, "On the accuracy of rf and image processing based hybrid localization for wireless capsule endoscopy", IEEE Wireless Communications and Networking Conference (WCNC), (2015).

[18] X. Li, Z. Lv and J. Hu, "Traffic management and forecasting system based on 3d gis", Cluster, Cloud and Grid Computing (CCGrid), 2015 15th IEEE/ACM International Symposium on, (2015). 991-998.

[19] S. Zhang and H. Jing, "Fast log-Gabor-based nonlocal means image denoising methods", Image Processing (ICIP), 2014 IEEE International Conference on IEEE, (2014), pp. 2724-2728.

[20] D. Jiang, Z. Xu and Z. Chen, "Joint time-frequency sparse estimation of large-scale network traffic", Computer Networks, 2011, 55(15): 3533-3547.

[21] J. Hu, Z. Gao and W. Pan, "Multiangle Social Network Recommendation Algorithms and Similarity Network Evaluation", Journal of Applied Mathematics, (2013).

[22] J. Hu and Z. Gao, "Modules identification in gene positive networks of hepatocellular carcinoma using Pearson agglomerative method and Pearson cohesion coupling modularity", Journal of Applied Mathematics, (2012).

[23] Z. Lv, A. Tek, F. D. Silva, "Game on, science-how video game technology may help biologists tackle visualization challenges", PloS one, vol. 8, no. 3, (2013), p. 57990.

\begin{abstract}
Author
Hu Dan, she received her M.S. degree in computer technology from Guizhou university in Guiyang, China. She is currently a lecturer in the College of Big Data and Information Engineering at Guizhou university. Her research interest is mainly in the area of Information theory and coding theory. She has published several research papers in scholarly journals in the above research areas and has participated in several books.
\end{abstract}

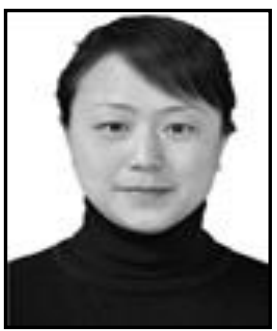


International Journal of Future Generation Communication and Networking Vol. 9, No.8, (2016) 